\title{
Comparative Adherence Trajectories of Oral Fingolimod and Injectable Disease Modifying Agents in Multiple Sclerosis
}

This article was published in the following Dove Press journal: Patient Preference and Adherence

\author{
Jagadeswara R Earla $\mathbb{D D}^{\prime}$ \\ George J Hutton ${ }^{2}$ \\ J Douglas Thornton (ID) \\ Hua Chen' \\ Michael L Johnson' \\ Rajender R Aparasu (ID) \\ 'Pharmaceutical Health Outcomes and \\ Policy, College of Pharmacy, University of \\ Houston, Houston, TX, USA; \\ ${ }^{2}$ Department of Neurology, Baylor \\ College of Medicine, Houston, TX, USA
}

Correspondence: Rajender R Aparasu Pharmaceutical Health Outcomes and Policy, College of Pharmacy, University of Houston, Texas Medical Center, 4849 Calhoun Road, Houston, TX77204-5047, USA

Tel + I 832-842-8374

Email rraparasu@uh.edu
Background: Oral fingolimod is convenient to use than injectable disease modifying agents (DMAs) in patients with multiple sclerosis (MS). However, the existing literature regarding the comparative adherence trajectories between oral fingolimod and injectable DMAs is limited.

Objective: To compare the adherence trajectories between oral DMA, fingolimod, and injectable DMAs in patients with MS.

Methods: A retrospective longitudinal study was conducted using adults ( $\geq 18$ years) with MS (ICD-9-CM: 340 and a DMA prescription) from the IBM MarketScan Commercial Claims and Encounters Database between 2010 and 2012. Patients were grouped into oral fingolimod or injectable DMA users based on the index DMA among patients with MS. The annual DMA adherence trajectories, based on the proportion of days covered (PDC), were examined using group-based trajectory modeling (GBTM) during the one-year follow-up period after treatment initiation. Multivariable multinomial logistic regression using stabilized inverse probability treatment weights (IPTW) was performed to assess the association between the DMA route of administration (Oral vs Injectable) and the adherence trajectory groups. The balance of covariates between oral and injectable DMAs before and after IPTW was checked against a standardized difference threshold of 0.25 .

Results: The study cohort consisted of 1,700 MS patients who were initiated with oral $(15.8 \%)$ or injectable (84.2\%) DMAs between 2010 and 2012. The adherence rates (PDC $\geq 0.8$ ) in oral fingolimod and injectable DMA users were found to be $64.7 \%$ and $50.8 \%$, respectively. The GBTM grouped individuals in the study cohort into three adherence trajectories - rapid discontinuers $(23.5 \%)$, complete adherers $(49.9 \%)$, and slow decliners $(26.6 \%)$. The multinomial logistic regression model with stabilized IPTW revealed that oral fingolimod users had higher odds to be a complete adherer (adjusted odds ratio [AOR]: 2.78, 95\% CI: $1.85-4.16$ ) or a slow discontinuer (AOR: 2.62, 95\% CI: $1.70-4.05$ ) than injectable DMA users.

Conclusions: Oral DMA fingolimod was associated with better adherence than injectable DMAs across group-based trajectories. Further research is warranted to evaluate the adherence trajectories with newer oral DMAs introduced in the last decade for MS.

Keywords: group based trajectory modelling, GBTM, adherence trajectory, multiple sclerosis, disease modifying agent, DMA, fingolimod, injectable DMA

\section{Plain Language Summary}

Comparative adherence evidence based on group-based trajectory modeling (GBTM) for oral and injectable disease modifying agents (DMAs) in MS is limited. The GBTM classified 
multiple sclerosis patients, based on adherence to DMAs, into rapid discontinuers $23.5 \%$, complete adherers $49.9 \%$, and slow decliners $26.6 \%$. Oral fingolimod was associated with better adherence trajectories than injectable DMAs. Oral fingolimod users had nearly three times higher odds to be in completely adherent or slow declining trajectory than injectable DMA users.

\section{Introduction}

Adherence to disease modifying agent (DMA) treatment is vital among patients with multiple sclerosis (MS) and is associated with positive clinical outcomes such as a reduction in relapses, progression of disability, and hospitalization. $^{1-3}$ Unfortunately, adherence to DMAs among patients with MS is often less than ideal ranging from $41 \%-88 \% .^{4-6}$ In addition, early discontinuation of DMA is very common in MS; $9 \%-20 \%$ of patients discontinue DMA within six months from the date of initiation. ${ }^{7}$ Non-adherence to DMA leads to serious negative consequences such as increased relapses, progression of disability, and higher healthcare resource utilization, and significantly impacts the quality of life..$^{2,3,5,7-9}$ Previous studies have found that adherence to DMAs is associated with factors related to the treatment route of administration, insurance coverage, patient and clinician. ${ }^{8,9}$

The route of administration is one of the important factors associated with treatment adherence. ${ }^{8}$ For almost two decades, injectable DMAs (interferon beta [1993] and glatiramer acetate [1996]) were the mainstay of treating patients with MS until the approval of the first oral DMA, fingolimod, in 2010. Injectable DMAs pose concerns such as fear of needles, the frequent need for administration, and adverse effects among patients with $\mathrm{MS}^{7}$ Adverse effects and lack of treatment effectiveness were seen as the primary reasons for non-adherence to injectable DMAs in MS. ${ }^{7,9}$ With the approval of fingolimod, the oral agent provided a good alternative for treating MS. Oral fingolimod, unlike injectable DMAs, is convenient to administer and can improve adherence. Further, oral DMAs are without the injection-related side effects. Oral fingolimod can also pose several safety issues and hence requires stringent monitoring. ${ }^{2,10}$ Therefore, the route of administration and associated administrative complexity can play a vital role in adherence to DMAs among patients with MS.

Although several real-world studies reported both oral and injectable DMAs to be tolerable, ${ }^{2,8,11,12}$ there is mixed evidence regarding the comparative adherence of oral versus injectable DMAs. ${ }^{8}$ Oral DMA users have shown comparable adherence relative to injectable DMAs and concluded that the route of administration of DMA was not a significant predictor of adherence. ${ }^{3,8,11,13-19}$ On the other hand, several other researchers also reported that oral DMAs showed better adherence than injectable DMAs ${ }^{1,20,21}$ and vice versa. ${ }^{15}$ Therefore, existing realworld evidence regarding the adherence between oral and injectable DMAs is mixed. ${ }^{1,8,11,13-23}$ In addition, these adherence studies ${ }^{1,8,13-23}$ assessed DMA adherence as a point estimate or a binary indicator using medication possession ratio (MPR) or proportion of days covered (PDC) over the study period rather than considering it as a dynamic process over time. ${ }^{24,25}$ Though these are wellaccepted measures to assess the adherence in observational studies; they overlook the valuable information about variation in the adherence over time, which can be better explained using alternate adherence modeling techniques such as trajectory modeling. ${ }^{24-27}$

The group-based trajectory modeling (GBTM) utilizes prescription-filling patterns over time to classify patients into different trajectory groups signifying their dynamic longitudinal adherence behavior. ${ }^{24-27}$ GBTM classifies the latent subgroups in a population with different adherence patterns. The subcategories and their adherence patterns can also be visually depicted graphically, allowing for a meaningful interpretation of adherence. ${ }^{27}$ Therefore, in this study, GBTM was preferred over conventional adherence methods to differentiate patients with different adherence patterns and to assess comparative adherence between oral and injectable DMAs. Nicholas et al assessed the adherence behavior among oral DMA users using GBTM; however, no comparison was made with injectable DMAs. $^{27}$ Hence, this study evaluated adherence trajectories of DMA users with MS and compared the trajectories between oral fingolimod and injectable DMAs. The study hypothesized that oral fingolimod users are associated with better adherence trajectories relative to injectable DMA users.

\section{Methods}

\section{Study Design and Data Source}

A retrospective longitudinal study was conducted using the IBM MarketScan Commercial Claims and Encounters data from 2010 to 2012. The IBM MarketScan, with more than 43.6 million commercially insured enrollees in the United States, provides a nationally representative sample of Americans with employer-provided health insurance. The enrollees are employees from large employers, 
government, and public organizations. The IBM MarketScan datasets include de-identified inpatient, outpatient, and pharmacy claims that can be linked for longitudinal analysis of health care utilization. The abovementioned files across the years were linked using the common enrollment identification number. ${ }^{28}$ This study was exempt from review by the Institutional Review Board at the University of Houston.

\section{Study Population}

The study population included adults ( $\geq 18$ years) diagnosed with MS and newly initiated oral fingolimod or injectable DMAs starting September 21, 2010 (after fingolimod's FDA approval) until December 31, 2011. Patients without DMA use during the six months prior to the index date were selected to exclude prevalent DMA users. Each patient was followed for medication utilization for at least one year from the date of the first DMA prescription, i.e., index date. Patients with MS diagnosis were identified based on the International Classification of Diseases, $9^{\text {th }}$ Revision, Clinical Modification (ICD-9-CM) code ' 340 . $\mathrm{XX}^{\prime}$ ' in diagnosis claims, and patients with DMA prescriptions were identified based on national drug codes (NDC) codes in pharmacy claims or the Healthcare Common Procedure Coding System (HCPCS) codes in outpatient or inpatient encounter files. The NDCs, universal drug identifier, of medications, were obtained from Redbook, and the HCPCS, standard healthcare procedure codes, were obtained from previous literature. ${ }^{8}$ Based on the first DMA prescription on the index date, patients were categorized into oral fingolimod or injectable users (interferon beta or glatiramer acetate). Combination DMA users and infusion DMA users were excluded as they are more severe patients and could lead to indication bias. The index prescription date was defined as the first DMA prescription fill date. Patients were required to have continuous enrollment with the health insurance plan during the 6 months prior and 12 months after the index prescription date. Continuous eligibility with the insurance plan ensures that patients' complete healthcare utilization during the study period was documented. The study design is illustrated in Supplementary Figure 1.

\section{Covariates}

The covariate selection for this study was guided by the conceptual framework of the Andersen Behavioral Model (ABM) of health care utilization. According to the ABM, healthcare utilization is contingent on three different sets of factors - predisposing, enabling, and need. ${ }^{29}$ Predisposing factors such as age group, gender, and region describe the predisposition of an individual to use healthcare service(s). Enabling factors such as employment status, type of health insurance plan, physician specialty coding, and prescription time period inform the ability or limitations of an individual to use health service(s). The physician specialty coding indicator was a predefined variable in MarketScan that flags if patients had highly differentiated $(\geq 70 \%)$ claims coded by specialty physicians. ${ }^{30}$ Need factors including prevalent comorbidities, Elixhauser score, MS severity score (MSSS), MS symptomatic medication, and health care utilization indicators, which explain the requirement of healthcare service(s) for an individual. ${ }^{29}$ Prevalent comorbidities among patients with MS were identified based on existing literature, ${ }^{31,32}$ and few additional comorbidities that were prevalent $(>15 \%)$ in the current study cohort were also identified using the clinical classification system (CCS) codes proposed by the Agency for Healthcare Research and Quality (AHRQ).$^{33}$ Elixhauser index score, a widely used surrogate measure of comorbidity burden in observational healthcare research, ${ }^{34}$ is a weighted score of selected comorbidities identified based on diagnoses claims in healthcare records. ${ }^{35}$ MSSS, a proxy measure of symptomatic burden or severity of MS, is a weighted score of selected MS-related symptoms or comorbidities identified using ICD-9-CM/HCPCS codes from diagnoses or procedure claims. A higher MSSS score indicates more severe or highly symptomatic MS, whereas a lower score indicates less severe or less symptomatic MS. ${ }^{36}$ The use of MS symptomatic medications to alleviate MS-related symptoms indicates neurological impairment. ${ }^{37}$ Healthcare utilization measures included baseline relapse, neurologist consultation, magnetic resonance imaging (MRI) test, and emergency department (ED) visit - MS-associated and nonMS-associated. The claims-based relapse measure is operationally defined as (i) an inpatient hospitalization, or (ii) an outpatient/emergency room encounter plus a steroid prescription within 30 days following the encounter. Successive relapses within the next 30 days after the initial relapse were consolidated into a single relapse episode. ${ }^{38}$ All the covariates were measured during the 6-months baseline period prior to the index prescription date.

\section{Statistical Analyses}

An a priori sample size calculation was conducted using PROC POWER in SAS with the effect size (odds ratio $2.01,95 \%$ confidence interval [CI] of 1.71-2.37) obtained 
from the previous literature ${ }^{20}$ at a pre-specified power of 0.8. This resulted in a sample size between 619 and 1,917. Characteristics of oral fingolimod and injectable DMA users were compared and assessed using descriptive statistical tests such as chi-square test for categorical variables and $t$-test for continuous variables.

The GBTM offers a longitudinal approach of measuring medication adherence based on the prescription-filling pattern over time to group different adherence trajectories. ${ }^{25}$ Patients within each trajectory group have a similar adherence pattern. ${ }^{39}$ These trajectories can be validated by assessing the outcomes of different trajectories. ${ }^{24,25}$ For this study, monthly DMA adherence for the 12 months during the follow-up period after the index prescription date was calculated using the proportion of days covered (PDC). PDC is preferred over Medication Possession Ratio (MPR) and other adherence estimates as PDC estimates are more conservative ${ }^{40,41}$ and are widely among observational studies. ${ }^{42}$ PDC is calculated as the ratio of "number of days of medication supply based on the DMA prescription/medication administration procedure records," identified using NDCs or HCPCS, to "the total number of days in the follow-up period," adjusting for overlaps. For injectable DMAs, the days of medication supply were decided based on the frequency of medication. For example, as glatiramer acetate is given weekly, one dose of glatiramer acetate is equal to 7 days of supply. As the objective is to measure the adherence with initial DMA, the adherence was measured until patients discontinue the initial DMA or switch to other DMA. Based on monthly PDC values, patients' monthly adherence status (adherent [PDC - $\geq 0.8$ ] or non-adherent [PDC - <0.8]) for 12 months was computed. ${ }^{43}$ Adherence was assessed during the one-year follow-up or until the patient was switched to another DMA. Using these 12 monthly adherence status indicators, adherence trajectories for the total cohort were modeled and plotted using GBTM (PROC TRAJ procedure in SAS). ${ }^{26}$ The selection of the number of trajectory groups and the appropriate shape (from first order to third order polynomial model) were decided based on the Bayesian information criterion (BIC) value and the subjective interpretability of the groups relative to one another. ${ }^{44,45}$ The sample size threshold in each trajectory group was set to $5 \%{ }^{46-48}$

In observational studies, unlike randomized control trials (RCTs), treatment groups tend to be systematically different from each other, and hence, can give biased treatment effect estimates due to selection bias. Propensity score (PS)-based methods became prominent as a solution to produce unbiased treatment estimates by balancing the differences between treatment groups through matching, stratification, weighting, or regression adjustment. The PS is the probability of receiving oral DMA compared to injectable DMA conditional on the available covariates and was computed using logistic regression. Among different PS methods, inverse probability treatment weighting (IPTW) is known to give marginal treatment estimates that are generalizable to the whole treatment population, unlike other methods. IPTW is the inverse of the probability of receiving respective treatments. The IPTWs were stabilized to avoid the issue of extreme weights and to produce treatment effect estimates with lesser variance. ${ }^{4-51}$ The PS-based IPTWs were estimated using PROC PSMATCH in SAS to create a pseudo population of oral fingolimod and injectable DMA users balanced on all the measured covariates. ${ }^{50}$ The covariates (predisposing, enabling, and need factors) for PS calculation were conceptualized based on the ABM, as explained before. The balance of covariates between oral fingolimod and injectable DMA groups was confirmed using the standardized differences with a satisfactory threshold value between -0.25 and 0.25 . $^{49}$

A multinomial logistic regression adjusting for stabilized IPTWs was performed to assess the association of the DMA route of administration (oral fingolimod versus injectable DMAs) with DMA adherence trajectory. The outcome variable was the adherence trajectory group assessed through GBTM. The primary independent variable was a binary indicator of oral fingolimod versus injectable DMA prescription; injectable DMA is considered as the reference category. Additional analysis of logistic regression adjusting for stabilized IPTWs was conducted to confirm the directionality of multinomial logistic regression findings. The dependent variable was a binary indicator of adherence status based on PDC $\geq 0.8$; non-adherence was considered as the reference category. All the statistical analyses were conducted using SAS 9.4 (SAS Institute, Cary, North Carolina) at a level of significance value of 0.05 .

\section{Results}

The study population consisted of 1,700 MS patients, among which $15.8 \%(\mathrm{n}=269)$ of patients started oral fingolimod, while the remaining $84.2 \%(n=1,431)$ started injectable DMAs. The cohort derivation chart is shown in Figure 1. Among injectable DMA users, $52.1 \%(\mathrm{n}=746)$ started interferon-beta, while the remainder $47.9 \%(n=685)$ started glatiramer acetate. The study population primarily consisted of females (79.0\%), young/middle-aged adults (18-44 years; 


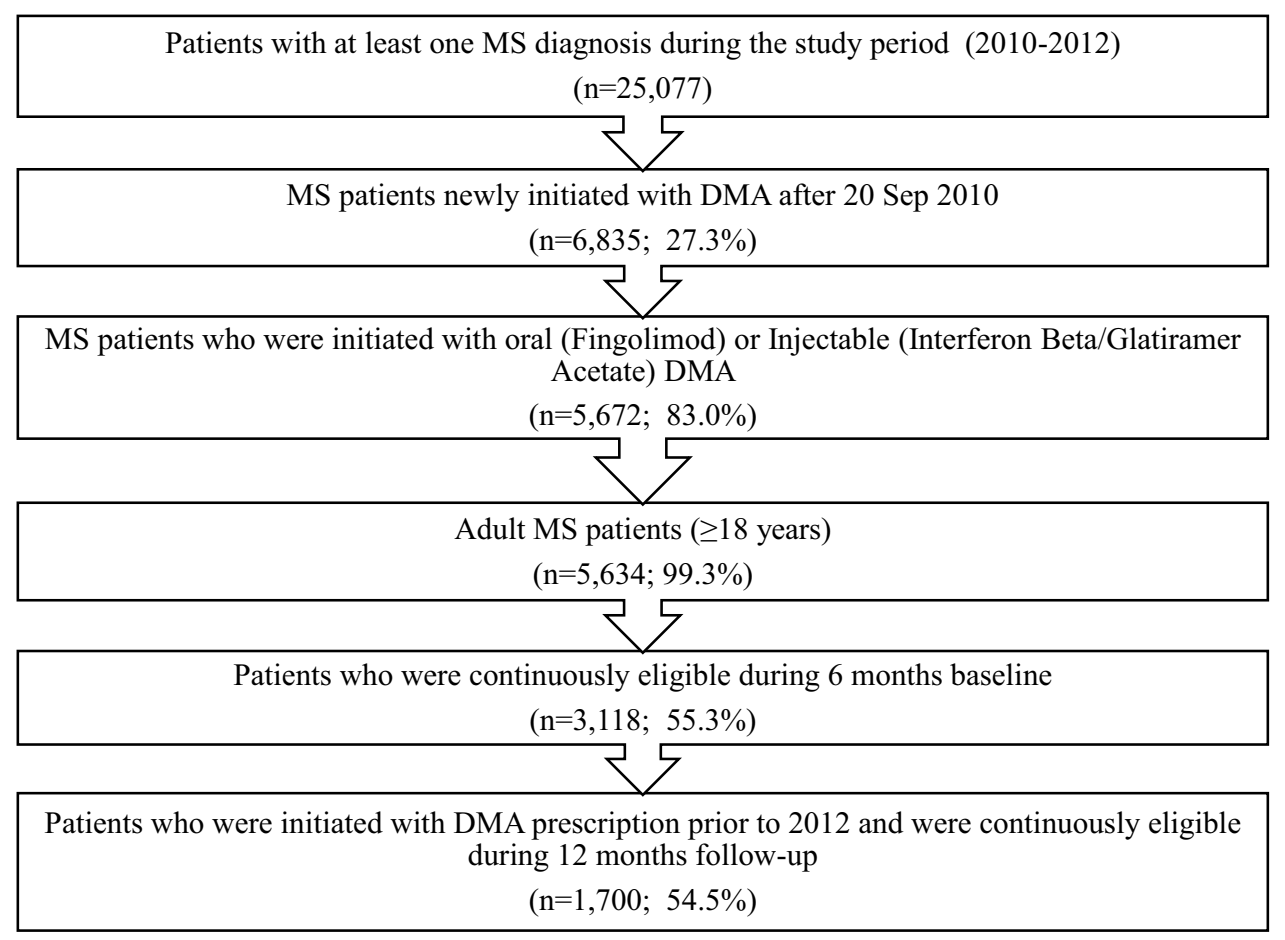

Figure I Flowchart For Study Cohort Derivation: IBM MarketScan 2010-2012.

$51.2 \%$ ), belonged to the South/West region (62.7\%), active full-time employees (80.9\%), and had Preferred Provider Organization (PPO) health insurance (58.9\%). Oral fingolimod and injectable DMA users significantly varied by the distribution of their predisposing (age group), enabling (employment status and prescription time period) and need factors (comorbidities, symptoms, symptomatic medication, and healthcare utilization). The characteristics of the total study cohort and by the route of administration of DMA are shown in Table 1.

Oral fingolimod users were significantly more adherent (annual PDC $\geq 0.8$ ) than injectable DMAs (Oral fingolimod [FIN] vs Injectable DMAs [INJ]: $64.7 \%$ vs $50.8 \%$ ). The GBTM classified patients with MS into three adherence trajectories - (i) Rapid discontinuers 23.5\% ( $\mathrm{n}=399)$, patients who discontinued DMAs early within 3-4 months after the initiation; (ii) Complete adherers $49.9 \%(\mathrm{n}=848)$, patients who were almost completely adherent to DMA throughout the follow-up period, (iii) Slow decliners $26.6 \%(\mathrm{n}=453)$, patients whose adherence gradually declined during the follow-up period. The model with three trajectories and quadratic polynomial form had better visual interpretability and lower BIC value than other models. (See Supplementary Figure 2). Adherence trajectories of patients with MS who received either oral fingolimod or injectable DMAs are shown in Figure 2.
Adherence trajectories of oral fingolimod and injectable DMA users are shown in Table 2. Patients across different trajectory groups significantly differed by the route of administration of DMA. A significantly higher number of oral fingolimod users were complete adherers compared to injectable DMA users (FIN vs INJ: $63.6 \%$ vs $47.3 \%$ ). On the other hand, the proportion of rapid discontinuers (FIN vs INJ: $11.5 \%$ vs $25.7 \%$ ) and slow decliners (FIN vs INJ: $24.9 \%$ vs $27.0 \%$ ) was lower in the oral fingolimod DMA group than in the injectable DMA group. Among, adherent patients $(n=901)$ based on annual adherence $(\mathrm{PDC} \geq 0.8), 92.6 \%(\mathrm{n}=834)$ fell in complete adherent trajectory and 7.4\% $(\mathrm{n}=67)$ fell in slow decliners trajectory, whereas, among, non-adherent $(\mathrm{PDC}<0.8)$ patients $(\mathrm{n}=799), 1.8 \%(\mathrm{n}=14)$ fell in complete adherer trajectory and $48.3 \%(n=386)$ fell in slow decliner trajectory. When modeled separately, oral fingolimod and injectable DMA users had similar adherence trajectories.

The IPTW balanced the covariate distribution between oral fingolimod and injectable DMA users. The propensity score distribution and the standard differences between oral fingolimod and injectable DMAs before and after IPTW are shown in Supplementary Figure 3, respectively. Stabilized IPTW-adjusted multinomial logistic regression findings revealed that relative to injectable DMA users, oral fingolimod users were nearly three times more 
Table I Characteristics of Oral and Injectable DMA Users with MS and the Standard Differences of Covariates Between Both Groups Before and After IPTW Weighting: IBM MarketScan 2010-2012

\begin{tabular}{|c|c|c|c|c|c|c|}
\hline \multirow[t]{3}{*}{ Characteristic } & \multicolumn{2}{|c|}{ Route of Administration } & \multirow{2}{*}{$\begin{array}{c}\text { Total } \\
(\mathrm{N}=\mathrm{I}, 700 \\
100.00 \%)\end{array}$} & \multirow{3}{*}{$\begin{array}{c}\text { p- } \\
\text { value }\end{array}$} & \multirow{3}{*}{$\begin{array}{l}\text { Standard } \\
\text { Difference } \\
\text { Before } \\
\text { IPTW }\end{array}$} & \multirow{3}{*}{$\begin{array}{c}\text { Standard } \\
\text { Difference } \\
\text { After } \\
\text { IPTW }\end{array}$} \\
\hline & $\begin{array}{l}\text { Oral } \\
\text { Fingolimod } \\
\text { Users } \\
(\mathrm{N}=269 ; \\
\text { 15.8\%) }\end{array}$ & $\begin{array}{l}\text { Injectable DMA Users } \\
\text { (N=I,43I; 84.2\%) }\end{array}$ & & & & \\
\hline & $\mathbf{N}(\%)$ & $\mathbf{N}(\%)$ & $\mathbf{N}(\%)$ & & & \\
\hline \multicolumn{7}{|l|}{ Predisposing variables } \\
\hline Age (in years, Mean \pm SD) & $45.6 \pm 10.0$ & $43.2 \pm 10.8$ & $43.6 \pm 10.7$ & 0.114 & & \\
\hline $\begin{array}{l}\text { Age Group (in years) } \\
\text { 18-44 } \\
45-64\end{array}$ & $\begin{array}{l}109(40.5 \%) \\
160(59.5 \%)\end{array}$ & $\begin{array}{l}761(53.2 \%) \\
670(46.8 \%)\end{array}$ & $\begin{array}{l}870(51.2 \%) \\
830(48.8 \%)\end{array}$ & 0.001 & -0.26 & 0.01 \\
\hline $\begin{array}{l}\text { Gender } \\
\text { Male } \\
\text { Female }\end{array}$ & $\begin{array}{l}55(20.4 \%) \\
214(79.6 \%)\end{array}$ & $\begin{array}{l}302(21.1 \%) \\
1,129(78.9 \%)\end{array}$ & $\begin{array}{l}357(21.0 \%) \\
1,343(79.0 \%)\end{array}$ & 0.808 & -0.02 & -0.04 \\
\hline $\begin{array}{l}\text { Region } \\
\text { Northeast } \\
\text { North central } \\
\text { South } \\
\text { West }\end{array}$ & $\begin{array}{l}37(13.8 \%) \\
64(23.8 \%) \\
99(36.8 \%) \\
69(25.6 \%)\end{array}$ & $\begin{array}{l}260(18.2 \%) \\
273(19.1 \%) \\
54 I(37.8 \%) \\
357(24.9 \%)\end{array}$ & $\begin{array}{l}297(17.5 \%) \\
337(19.8 \%) \\
640(37.6 \%) \\
426(25.1 \%)\end{array}$ & 0.160 & & \\
\hline $\begin{array}{l}\text { Region } \\
\text { Northeast/North central } \\
\text { South/West }\end{array}$ & $\begin{array}{l}101(37.6 \%) \\
168(62.5 \%)\end{array}$ & $\begin{array}{l}533(37.3 \%) \\
898(62.7 \%)\end{array}$ & $\begin{array}{l}634(37.3 \%) \\
1,066(62.7 \%)\end{array}$ & 0.926 & 0.01 & -0.15 \\
\hline \multicolumn{7}{|l|}{ Enabling Variables } \\
\hline $\begin{array}{l}\text { Employment Status } \\
\text { Active Full Time } \\
\text { Others* }\end{array}$ & $\begin{array}{l}203(75.5 \%) \\
66(24.5 \%)\end{array}$ & $\begin{array}{l}\text { I, } 173(82.0 \%) \\
258(18.0 \%)\end{array}$ & $\begin{array}{l}1,376(80.9 \%) \\
324(19.1 \%)\end{array}$ & 0.029 & -0.16 & 0.00 \\
\hline $\begin{array}{l}\text { Plan Indicator } \\
\text { Others (HMO, POS, EPO, POS with capitation, } \\
\text { CDHP, HDHP) } \\
\text { Preferred Provider Organization (PPO) }\end{array}$ & $\begin{array}{l}108(40.2 \%) \\
161(59.8 \%)\end{array}$ & $\begin{array}{l}591(41.3 \%) \\
840(58.7 \%)\end{array}$ & $\begin{array}{l}699(41.1 \%) \\
1,001(58.9 \%)\end{array}$ & 0.725 & -0.02 & 0.10 \\
\hline $\begin{array}{l}\text { Physician Specialty Coding Flag } \\
<70 \% \text { of outpatient physician records have } \\
\text { specialty indicated } \\
70 \% \text { or more of outpatient physician records } \\
\text { have specialty indicated }\end{array}$ & $\begin{array}{l}10(3.7 \%) \\
259(96.3 \%)\end{array}$ & $\begin{array}{l}55(3.8 \%) \\
1,376(96.2 \%)\end{array}$ & $\begin{array}{l}65(3.8 \%) \\
1,635(96.2 \%)\end{array}$ & 0.921 & & \\
\hline $\begin{array}{l}\text { Date Year Incurred } \\
2010 \\
2011\end{array}$ & $\begin{array}{l}22(8.2 \%) \\
247(91.8 \%)\end{array}$ & $\begin{array}{l}32 \mathrm{I}(22.4 \%) \\
\mathrm{I}, \mathrm{I} 10 \text { (77.6\%) }\end{array}$ & $\begin{array}{l}343(20.2 \%) \\
1,357(79.8 \%)\end{array}$ & $<0.050$ & -0.40 & -0.04 \\
\hline \multicolumn{7}{|l|}{ Need Factors } \\
\hline Mean Elixhauser Score $[$ Mean \pm SD] & $1.8 \pm 4.1$ & $2.0 \pm 4.1$ & $2.0 \pm 4.1$ & 0.013 & & \\
\hline $\begin{array}{l}\text { Selected AHRQ CCS comorbidities that } \\
\text { are prevalent in MS Patients } \\
\text { Infections }\end{array}$ & $68(25.3 \%)$ & $281(19.6 \%)$ & $349(20.5 \%)$ & 0.036 & -0.14 & 0.06 \\
\hline
\end{tabular}

(Continued) 
Table I (Continued).

\begin{tabular}{|c|c|c|c|c|c|c|}
\hline \multirow[t]{3}{*}{ Characteristic } & \multicolumn{2}{|c|}{ Route of Administration } & \multirow{2}{*}{$\begin{array}{c}\text { Total } \\
(\mathrm{N}=\mathrm{I}, 700 ; \\
\text { 100.00\%) }\end{array}$} & \multirow{3}{*}{$\begin{array}{c}\text { p- } \\
\text { value }\end{array}$} & \multirow{3}{*}{$\begin{array}{c}\text { Standard } \\
\text { Difference } \\
\text { Before } \\
\text { IPTW }\end{array}$} & \multirow{3}{*}{$\begin{array}{c}\text { Standard } \\
\text { Difference } \\
\text { After } \\
\text { IPTW }\end{array}$} \\
\hline & $\begin{array}{l}\text { Oral } \\
\text { Fingolimod } \\
\text { Users } \\
\text { (N=269; } \\
\text { 15.8\%) }\end{array}$ & $\begin{array}{l}\text { Injectable DMA Users } \\
(\mathrm{N}=1,43 \text { I; 84.2\%) }\end{array}$ & & & & \\
\hline & $\mathbf{N}(\%)$ & $\mathbf{N}(\%)$ & $\mathbf{N}(\%)$ & & & \\
\hline Cancer & $46(17.1 \%)$ & $185(12.9 \%)$ & $231(13.6 \%)$ & 0.067 & -0.12 & 0.03 \\
\hline $\begin{array}{l}\text { Metabolic Disorders } \\
\text { Thyroid Disorders } \\
\text { Diabetes Mellitus } \\
\text { Nutritional Deficiencies } \\
\text { Lipid Disorders }\end{array}$ & $\begin{array}{l}28(10.4 \%) \\
21(7.8 \%) \\
15(5.6 \%) \\
33(12.3 \%)\end{array}$ & $\begin{array}{l}136(9.5 \%) \\
130(9.1 \%) \\
128(8.9 \%) \\
197(13.8 \%)\end{array}$ & $\begin{array}{l}164(9.7 \%) \\
151(8.9 \%) \\
143(8.4 \%) \\
230(13.5 \%)\end{array}$ & $\begin{array}{l}0.645 \\
0.499 \\
0.068 \\
0.510\end{array}$ & $\begin{array}{l}-0.03 \\
0.05 \\
0.13 \\
0.04\end{array}$ & $\begin{array}{l}0.03 \\
0.09 \\
-0.09 \\
0.10\end{array}$ \\
\hline $\begin{array}{l}\text { Mental Illness } \\
\text { Anxiety } \\
\text { Bipolar Disorders } \\
\text { Depression }\end{array}$ & $\begin{array}{l}12(4.5 \%) \\
7(2.6 \%) \\
33(12.3 \%)\end{array}$ & $\begin{array}{l}94(6.6 \%) \\
28(2.0 \%) \\
138(9.6 \%)\end{array}$ & $\begin{array}{l}106(6.2 \%) \\
35(2.1 \%) \\
17 \mid(10.1 \%)\end{array}$ & $\begin{array}{l}0.190 \\
0.494 \\
0.189\end{array}$ & $\begin{array}{l}0.09 \\
-0.04 \\
-0.08\end{array}$ & $\begin{array}{l}-0.07 \\
0.02 \\
0.11\end{array}$ \\
\hline $\begin{array}{l}\text { Neurological Disorders } \\
\text { Paralysis } \\
\text { Epilepsy } \\
\text { Convulsions } \\
\text { Migraine headache } \\
\text { Other Headaches } \\
\text { Eye Disorders } \\
\text { Ear Disorders } \\
\text { Other Neurological Disorders } \dagger\end{array}$ & $\begin{array}{l}15(5.6 \%) \\
4(1.5 \%) \\
5(1.9 \%) \\
12(4.5 \%) \\
25(9.3 \%) \\
121(45.0 \%) \\
22(8.2 \%) \\
102(37.9 \%)\end{array}$ & $\begin{array}{l}54(3.8 \%) \\
20(1.4 \%) \\
35(2.5 \%) \\
127(8.9 \%) \\
229(16.0 \%) \\
393(27.5 \%) \\
206(14.4 \%) \\
786(54.9 \%)\end{array}$ & $\begin{array}{l}69(4.1 \%) \\
24(1.4 \%) \\
40(2.4 \%) \\
139(8.2 \%) \\
254(14.9 \%) \\
514(30.2 \%) \\
228(13.4 \%) \\
888(52.2 \%)\end{array}$ & $\begin{array}{l}0.169 \\
0.909 \\
0.560 \\
0.015 \\
0.005 \\
<0.050 \\
0.006 \\
<0.050\end{array}$ & $\begin{array}{l}-0.09 \\
-0.01 \\
0.04 \\
0.18 \\
0.20 \\
-0.37 \\
0.20 \\
0.35\end{array}$ & $\begin{array}{l}0.06 \\
0.06 \\
0.04 \\
0.01 \\
0.14 \\
0.05 \\
-0.09 \\
0.14\end{array}$ \\
\hline $\begin{array}{l}\text { Circulatory/Vascular Disorders } \\
\text { Hypertension } \\
\text { Heart Diseases } \\
\text { Cerebrovascular Disease }\end{array}$ & $\begin{array}{l}38(14.1 \%) \\
62(23.1 \%) \\
12(4.5 \%)\end{array}$ & $\begin{array}{l}219(15.3 \%) \\
208(14.5 \%) \\
155(10.8 \%)\end{array}$ & $\begin{array}{l}257(15.1 \%) \\
270(15.9 \%) \\
167(9.8 \%)\end{array}$ & $\begin{array}{l}0.621 \\
0.001 \\
0.001\end{array}$ & $\begin{array}{l}0.03 \\
-0.22 \\
0.24\end{array}$ & $\begin{array}{l}0.11 \\
0.10 \\
-0.10\end{array}$ \\
\hline $\begin{array}{l}\text { Respiratory Disorders } \\
\text { Chronic Lung Disease (CLD) }\end{array}$ & $8(3.0 \%)$ & $24(1.7 \%)$ & $32(1.9 \%)$ & 0.151 & -0.04 & 0.12 \\
\hline $\begin{array}{l}\text { Gastrointestinal Disorders } \\
\text { Liver Diseases }\end{array}$ & $4(1.49 \%)$ & $35(2.5 \%)$ & $39(2.3 \%)$ & 0.335 & 0.07 & -0.03 \\
\hline $\begin{array}{l}\text { Genitourinary Disorders } \\
\text { Diseases of the Urinary System }\end{array}$ & 71 (26.4\%) & $266(18.6 \%)$ & 337 (19.8\%) & 0.003 & -0.19 & 0.11 \\
\hline $\begin{array}{l}\text { Complications related to Pregnancyl } \\
\text { Childbirth }\end{array}$ & $5(1.9 \%)$ & $76(5.3 \%)$ & $81(4.8 \%)$ & 0.015 & 0.19 & 0.11 \\
\hline $\begin{array}{l}\text { Diseases of the skin and subcutaneous } \\
\text { tissue }\end{array}$ & 53 (19.7\%) & 175 (I2.2\%) & 228 (13.4\%) & 0.001 & -0.21 & 0.08 \\
\hline $\begin{array}{l}\text { Musculoskeletal Disorders } \\
\text { Non-traumatic joint disorders } \\
\text { Spondylosis, intervertebral disc disorders, other } \\
\text { back problems }\end{array}$ & $\begin{array}{l}51(19.0 \%) \\
83(30.9 \%)\end{array}$ & $\begin{array}{l}252(17.6 \%) \\
557(38.9 \%)\end{array}$ & $\begin{array}{l}303(17.8 \%) \\
640(37.7 \%)\end{array}$ & $\begin{array}{l}0.596 \\
0.012\end{array}$ & $\begin{array}{l}-0.03 \\
0.17\end{array}$ & $\begin{array}{l}0.06 \\
0.04\end{array}$ \\
\hline $\begin{array}{l}\text { Other connective tissue diseases (including } \\
\text { Fibromyalgia) }\end{array}$ & $58(21.6 \%)$ & $423(29.6 \%)$ & $48 I$ (28.3\%) & 0.008 & 0.18 & 0.03 \\
\hline
\end{tabular}


Table I (Continued).

\begin{tabular}{|c|c|c|c|c|c|c|}
\hline \multirow[t]{3}{*}{ Characteristic } & \multicolumn{2}{|c|}{ Route of Administration } & \multirow{2}{*}{$\begin{array}{c}\text { Total } \\
(\mathrm{N}=\mathrm{I}, 700 ; \\
\text { 100.00\%) }\end{array}$} & \multirow{3}{*}{$\begin{array}{c}\text { p- } \\
\text { value }\end{array}$} & \multirow{3}{*}{$\begin{array}{c}\text { Standard } \\
\text { Difference } \\
\text { Before } \\
\text { IPTW }\end{array}$} & \multirow{3}{*}{$\begin{array}{c}\text { Standard } \\
\text { Difference } \\
\text { After } \\
\text { IPTW }\end{array}$} \\
\hline & $\begin{array}{c}\text { Oral } \\
\text { Fingolimod } \\
\text { Users } \\
\text { (N=269; } \\
\text { 15.8\%) }\end{array}$ & $\begin{array}{l}\text { Injectable DMA Users } \\
\qquad(\mathrm{N}=1,431 ; 84.2 \%)\end{array}$ & & & & \\
\hline & $\mathbf{N}(\%)$ & $\mathbf{N}(\%)$ & $\mathbf{N}(\%)$ & & & \\
\hline \multicolumn{7}{|l|}{ III-defined Conditions } \\
\hline Nausea, vomiting/abdominal Pain & $26(9.7 \%)$ & $162(11.3 \%)$ & $188(11.1 \%)$ & 0.427 & 0.05 & -0.02 \\
\hline MS related Symptoms/Mobility Impairment & 161 (59.9\%) & $902(63.0 \%)$ & $1,063(62.5 \%)$ & 0.323 & & \\
\hline MS related symptoms & $703(58.7 \%)$ & $896(62.6 \%)$ & I,054 (62.0\%) & 0.229 & & \\
\hline Bladder/bowel symptoms & $31(11.5 \%)$ & $105(7.3 \%)$ & $136(8.0 \%)$ & 0.020 & & \\
\hline Brainstem symptoms & $23(8.6 \%)$ & $222(15.5 \%)$ & $245(14.4 \%)$ & 0.003 & & \\
\hline Cerebellar symptoms & $34(12.6 \%)$ & $192(13.4 \%)$ & $226(13.3 \%)$ & 0.730 & & \\
\hline Cerebral symptoms/cognitive Impairment & $8(3.0 \%)$ & $48(3.4 \%)$ & $56(3.3 \%)$ & 0.749 & & \\
\hline Difficulty walking/gait problems & $23(8.6 \%)$ & $113(7.9 \%)$ & $136(8.0 \%)$ & 0.717 & & \\
\hline General symptoms & $35(13.0 \%)$ & 257 (18.0\%) & $292(17.2 \%)$ & 0.048 & & \\
\hline Pyramidal symptoms & $26(9.7 \%)$ & $113(7.9 \%)$ & $139(8.2 \%)$ & 0.331 & & \\
\hline Sensory symptoms & $2(0.7 \%)$ & $27(1.9 \%)$ & $29(1.7 \%)$ & 0.184 & & \\
\hline Speech symptoms & $39(14.5 \%)$ & $413(28.9 \%)$ & $452(26.6 \%)$ & $<0.050$ & & \\
\hline Visual symptoms & $54(20.1 \%)$ & $215(15.0 \%)$ & $269(15.8 \%)$ & 0.037 & & \\
\hline $\begin{array}{l}\text { Mobility Impairment - Durable Medical } \\
\text { Equipment (DME) }\end{array}$ & $17(6.3 \%)$ & $55(3.84 \%)$ & $72(4.2 \%)$ & 0.064 & & \\
\hline MS Severity Score [MSSS, Mean \pm SD] & $1.5 \pm 1.8$ & $1.6 \pm 1.9$ & $1.6 \pm 1.9$ & 0.896 & & \\
\hline \multicolumn{7}{|l|}{ MS Symptomatic Medication } \\
\hline Analgesics & 209 (77.7\%) & I,I $122(78.4 \%)$ & I,331 (78.3\%) & 0.795 & 0.02 & -0.10 \\
\hline Anticonvulsants & $68(25.3 \%)$ & $318(22.2 \%)$ & $386(22.7 \%)$ & 0.272 & -0.05 & 0.09 \\
\hline Antidepressants & 161 (59.9\%) & $819(57.2 \%)$ & $980(57.7 \%)$ & 0.425 & -0.31 & 0.13 \\
\hline Bladder Dysfunction Drugs & $83(30.9 \%)$ & $256(17.9 \%)$ & $339(19.9 \%)$ & $<0.050$ & -0.04 & 0.01 \\
\hline Cognition drugs & $7(2.6 \%)$ & $39(2.7 \%)$ & $46(2.7 \%)$ & 0.909 & -0.26 & 0.14 \\
\hline Erectile dysfunction drugs & $7(2.6 \%)$ & $59(4.1 \%)$ & $66(3.9 \%)$ & 0.236 & -0.42 & 0.06 \\
\hline Fatigue Drugs & $80(29.7 \%)$ & $398(27.8 \%)$ & $478(28.1 \%)$ & 0.519 & 0.08 & -0.04 \\
\hline Impaired walking drugs & $65(24.2 \%)$ & $129(9.0 \%)$ & $194(11.4 \%)$ & $<0.050$ & -0.07 & -0.08 \\
\hline Spasticity Drugs & 192 (71.4\%) & $846(59.1 \%)$ & $1,038(61.1 \%)$ & 0.0001 & 0.01 & 0.09 \\
\hline \multicolumn{7}{|l|}{ Healthcare Utilization } \\
\hline Relapse & $42(15.6 \%)$ & $267(18.7 \%)$ & $309(18.2 \%)$ & 0.235 & 0.08 & 0.05 \\
\hline Neurologist consultation & $143(53.2 \%)$ & $610(42.6 \%)$ & $753(44.3 \%)$ & 0.001 & -0.21 & 0.00 \\
\hline Magnetic resonance imaging (MRI) & $5(1.9 \%)$ & $60(4.2 \%)$ & $65(3.8 \%)$ & 0.067 & 0.14 & 0.03 \\
\hline Emergency department (ED) visits & & & & 0.025 & 0.15 & 0.18 \\
\hline No ED visit & $221(82.2 \%)$ & I,086 (75.9\%) & I,307 (76.9\%) & & & \\
\hline ED Visit & 48 (17.8\%) & $345(24.1 \%)$ & $393(23.1 \%)$ & & & \\
\hline
\end{tabular}

Notes: * Other employment status include part-time/seasonal, early retiree, long-term disabled, etc. $\dagger$ Other neurological disorders include cerebral degeneration (unspecified), Parkinson's disease, Huntington's chorea, other choreas, neuroleptic malignant syndrome, spinocerebellar disease, trigeminal nerve disorders, other retinal disorders, other demyelinating diseases of central nervous system (neuromyelitis optica, Schilder's disease, acute transverse myelitis, other demyelinating diseases of central nervous system), epilepsy and recurrent seizures, anoxic brain damage, encephalopathy, convulsions and aphasia Standard differences for certain covariates was not shown as they were not considered for propensity score weighting due to multicollinearity.

Abbreviations: MS, multiple sclerosis; DMA, disease modifying agent; IPTW, inverse probability treatment weighting; SD, standard deviation; HMO, health maintenance organization; POS, point-of-service; PPO, preferred provider organization; EPO, exclusive provider organization; CDHP, consumer-directed health plan; HDHP, high deductible health plan.

likely to be in the completely adherent trajectory (Adjusted odds ratio [aOR]: 2.78, 95\% Confidence Interval [CI]: 1.85-4.16) or slow declining trajectory (aOR: $2.62,95 \%$
CI: 1.70-4.05). Results of the multinomial logistic regression assessing the association of adherence trajectory with the route of administration are shown in Table 3 . 


\section{DMA Adherence Trajectories Among Patients with Multiple Sclerosis}

3 groups, 2 order

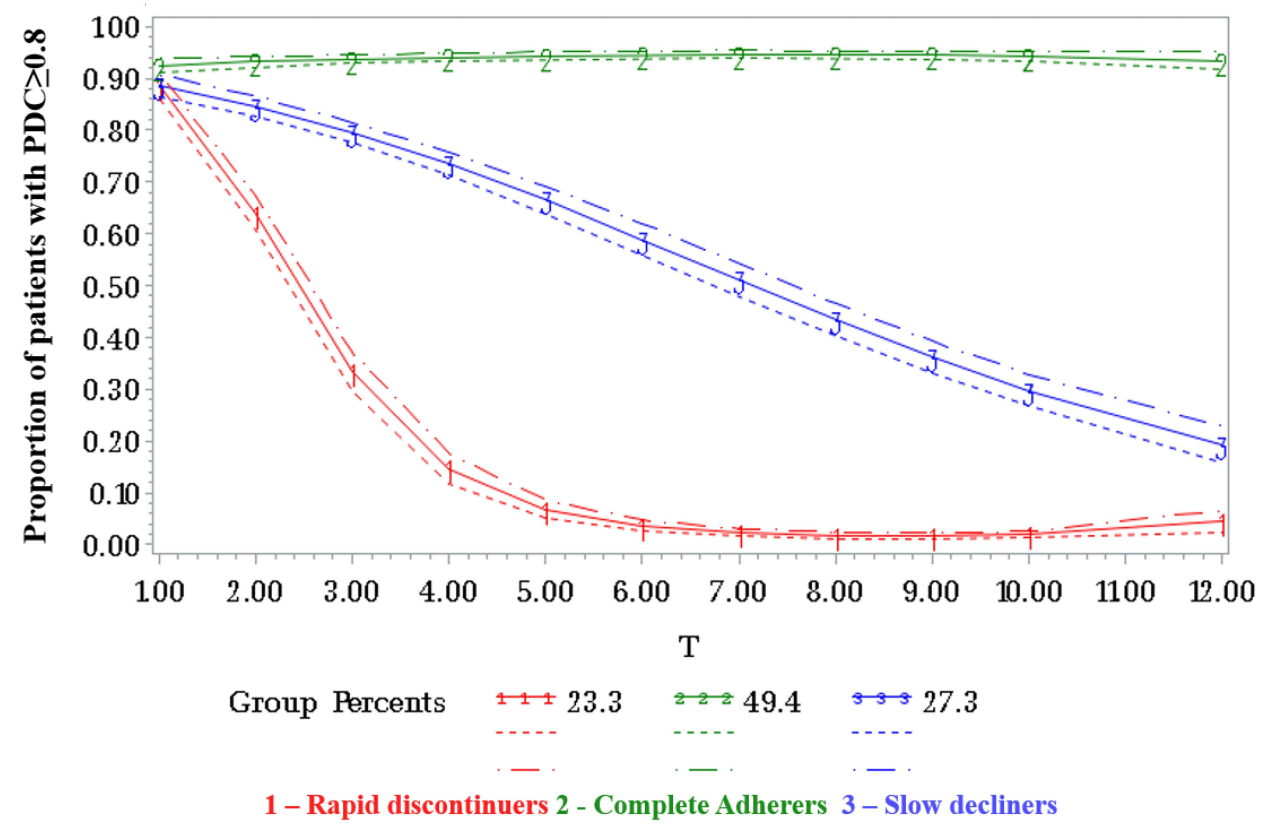

Figure 2 Adherence Trajectories of Patients with MS who received Oral Fingolimod or Injectable DMAs. Rapid discontinuers: Patients who discontinued DMAs early within 3-4 months after the initiation. Complete adherers: Patients who were almost completely adherent to DMA throughout the follow-up period. Slow decliners: Patients whose adherence gradually declined during the follow-up period.

Abbreviations: MS, multiple sclerosis; DMA, disease modifying agent.

Confirming the findings from multinomial logistic regression, additional analyses of stabilized IPTW-adjusted logistic regression revealed that oral fingolimod users were 1.43 times more likely to be adherent, regardless of the adherence trajectory, compared to injectable DMA users (aOR: 1.43, 95\% CI: 1.11-1.87).

\section{Discussion}

This study evaluated the DMA adherence in patients with MS using the GBTM and compared the adherence trajectories between oral fingolimod and injectable DMA users with MS. The GBTM provides longitudinal adherence trajectories based on the prescription-filling pattern of patients over time. ${ }^{25}$ Each trajectory group has a similar adherence pattern distinct from other trajectory groups. ${ }^{39}$ Overall, this study found three adherence trajectories involving complete adherers, slow decliners, and rapid discontinuers in the MS cohort. About half of the study sample were complete adherers irrespective of the dosage form. Among non-adherent patients, patients were almost equally distributed between rapid discontinuers and slow decliners. Previous studies found that adherence to DMAs among patients with MS ranged from $41 \%-88 \% .^{4-6}$ This study found three different and distinct adherence trajectories for DMAs. These findings can help understand

Table 2 Adherence Trajectories Between Oral Fingolimod and Injectable DMA Users in Patients with MS

\begin{tabular}{|c|c|c|c|c|}
\hline \multirow{2}{*}{$\begin{array}{c}\text { Adherence Trajectory Group } \\
\text { (Mean PDC } \pm \text { SD) }\end{array}$} & \multicolumn{2}{|c|}{ Route of Administration } & \multirow[b]{2}{*}{$\begin{array}{c}\text { Total } \\
N=1,700(100.00 \%)\end{array}$} & \multirow[b]{2}{*}{ p value } \\
\hline & $\begin{array}{l}\text { Oral Fingolimod } \\
\mathrm{N}=269(15.8 \%)\end{array}$ & $\begin{array}{l}\text { Injectable DMAs* } \\
\mathrm{N}=1,43 \mid \text { ( } 81.2 \%)\end{array}$ & & \\
\hline $\begin{array}{l}\text { I. Rapid discontinuers }(0.24 \pm 0.13) \\
\text { 2. Complete adherers }(0.96 \pm 0.05) \\
\text { 3. Slow decliners }(0.64 \pm 0.14)\end{array}$ & $\begin{array}{l}31(11.5 \%) \\
17 \mid(63.6 \%) \\
67(24.9 \%)\end{array}$ & $\begin{array}{l}368(25.7 \%) \\
677(47.3 \%) \\
386(27.0 \%)\end{array}$ & $\begin{array}{l}399(23.5 \%) \\
848(49.9 \%) \\
453(26.6 \%)\end{array}$ & $<0.001$ \\
\hline
\end{tabular}

Note: *Injectable DMAs included interferon beta and glatiramer acetate.

Abbreviations: MS, multiple sclerosis; DMA, disease modifying agent; PDC, proportion of days covered; SD, standard deviation. 
Table 3 Findings of IPTW Weighted Multinomial Logistic Regression

\begin{tabular}{|l|c|c|c|}
\hline \multicolumn{3}{|c|}{$\begin{array}{c}\text { Association of DMA Adherence Trajectory with DMA Route of Administration } \\
\text { (Reference Trajectory Group: Group I, Rapid Discontinuers) }\end{array}$} \\
\hline $\begin{array}{l}\text { DMA Route of } \\
\text { Administration }\end{array}$ & Sample & \multicolumn{2}{|c|}{ Adherence Trajectory Group } \\
\cline { 2 - 4 } & & $\begin{array}{c}\text { Group 2 } \\
\text { (Complete Adherers) } \\
\text { (Slow Decliners) }\end{array}$ \\
\cline { 3 - 4 } & & aOR (95\% Cl) & aOR (95\% Cl) \\
\hline $\begin{array}{l}\text { Injectable DMAs } \\
\text { Oral Fingolimod }\end{array}$ & Reference & $\begin{array}{l}\text { Refence } \\
2.62(1.70-4.05)\end{array}$ \\
\hline
\end{tabular}

Abbreviations: MS, multiple sclerosis; DMA, disease modifying agent; aOR, adjusted odds ratio; Cl, confidence interval; IPTW, inverse probability treatment weights.

adherence patterns over time, and the factors influencing these trajectories to improve the quality of care in MS. In addition, there can be a possibility of different intervention strategies to address the issue of non-adherence based on these trajectories. Most importantly, this study found that oral fingolimod users were associated with better adherence trajectories than injectable DMA users across groupbased trajectories.

Evaluation of trajectories revealed that oral fingolimod users had nearly three times higher odds to be a complete adherer or a slow discontinuer. Additional analyses also confirmed that oral fingolimod users are more adherent than injectable DMA users. This was the first study that compared adherence trajectories between oral and injectable DMA users; therefore, a comparison of current study findings in the context of previous evidence is not possible. However, some previous evidence based on conventional adherence measures indicated that oral fingolimod is associated with better adherence than injectable DMAs. ${ }^{1,20,21}$ This study showed that oral fingolimod users are more adherent and further suggests that they are associated with better adherence trajectories than injectable DMA users. The association between the route of administration and high adherence trajectories also showed a consistent pattern. However, more studies with newer oral DMAs (teriflunomide, dimethyl fumarate, siponimod, and cladribine) are needed to establish the evidence on comparative adherence between oral and injectable DMAs There is also a need to compare the adherence among the newer oral DMAs introduced in the last decade among individuals with MS.

As MS is a chronic and progressive disease, measuring DMA adherence through GBTM would significantly inform time-related changes in disease progression and its impact on DMA adherence. Adherence to DMAs is essential among patients with MS to reduce relapses and show favorable health outcomes. The GBTM can help to better characterize patients with similar DMA adherence patterns than the conventional adherence measures (PDC or MPR, etc.). More research is needed involving the GBTM with newer DMAs in the last decade in furthering the adherence research in MS. These trajectories can also help clinicians and patients to see which DMAs facilitate better adherence and to identify patient groups with barriers to continually adhere to DMAs. Tailored intervention strategies can also be developed, at the group level, for different trajectory patterns to improve adherence. Therefore, this GBTM-based adherence study showed that the first oral DMA fingolimod had better adherence trajectories than injectable DMAs with significant clinical as well as policy implications for patient care in MS.

\section{Strengths and Limitations}

This is the first study that addressed the issue of comparative adherence between oral and injectable DMAs using GBTM after the introduction of the first oral DMA. Another important strength of this study is that it accounted for a variety of clinical variables such as comorbidities/comorbidity burden, MS severity, symptomatic medications, and healthcare utilization. This study used IPTWs to provide minimally biased population-level marginal effects without limiting the generalizability based on the observed sample. ${ }^{49,50}$ However, this study is not without limitations. Using adherence trajectories may cause interpretation difficulties with more number of trajectory groups. In addition, if both treatment groups or medications show different trajectory patterns when modeled separately, it indicates that those treatment groups are not 
comparable using trajectory groups. Therefore, caution needs to be exercised while using GBTM modeling for comparative adherence research. It should also be acknowledged that there is an issue of unmeasured confounding. The data source lacked information pertaining to certain predisposing (race/ethnicity), enabling (income and physician-related), and need (MS phenotype, MS disability/EDSS score, MRI lesions, etc.) factors that could have strengthened the study results. However, the use of MSSS and MS symptomatic medication might have partly addressed this concern by serving as a proxy for MS severity. ${ }^{36,52}$ This study calculated adherence based on the prescription fill data from administrative claims data, which does not necessarily indicate medication administration/actual medication utilization by patients. Further, this study did not include other newer oral agents such as dimethyl fumarate, teriflunomide, siponimod, and cladribine. Therefore, the above limitations should be kept in mind while interpreting and generalizing the study findings.

\section{Conclusions}

This study compared the adherence trajectories of oral fingolimod and injectable DMAs after the introduction of the first oral DMA. The GBTM found that oral fingolimod was associated with better adherence trajectories than injectable DMAs. Oral fingolimod users were nearly three times more likely to be a complete adherer or a slow discontinuer. The association between the route of administration and high adherence trajectories showed a consistent pattern. Since MS is a chronic and progressive disease, adherence to DMAs is essential among patients with MS to reduce relapses and show favorable health outcomes. Therefore, further research is needed to evaluate adherence trajectories with other newer oral DMAs introduced in the last decade. In addition, research regarding comparative adherence trajectories between oral and injectable DMAs would also be of significant relevance for MS practitioners and patients with MS. These study findings can help clinicians and patients in understanding the adherence trajectories of oral fingolimod and assist them in making better treatment decisions for patients with MS.

\section{Abbreviations}

MS, multiple sclerosis; DMA, disease modifying agent; PDC, proportion of days covered; MPR, Medication Possession Ratio; GBTM, group based trajectory modeling;
ICD-9-CM, International Classification of Diseases, Ninth Revision, Clinical Modification; NDC, National Drug Code; HCPCS, The Healthcare Common Procedure Coding System; ABM, The Andersen Behavioral Model; CCS, Clinical Classification System; AHRQ, Agency for Healthcare Research and Quality; MSSS, Multiple Sclerosis Severity Score; MRI, Magnetic Resonance Imaging; ED, Emergency Department; BIC, Bayesian Information Criterion; RCT, Randomized Control Trials; PS, Propensity Score; IPTW, inverse probability treatment weighting; FIN, Fingolimod; INJ, Injectable DMAs; AOR, Adjusted Odds Ratio; SD, Standard Deviation; CI, Confidence Interval; EDSS, Expanded Disability Status Score; HMO, Health Maintenance Organization; POS, Point-of-service; PPO, Preferred Provider Organization; EPO, Exclusive Provider Organization; CDHP, Consumer Directed Health Plan; HDHP, High Deductible Health Plan; CLD, Chronic Lung Disease; DME, Durable Medical Equipment.

\section{Acknowledgments}

A part of the study findings was presented as an abstract at the International Society for Pharmacoeconomics and Outcomes (ISPOR) $25^{\text {th }}$ Annual International Meeting Research Abstracts, May 2020, Value in Health 23, S279.

\section{Funding}

This is not a funded/sponsored study.

\section{Disclosure}

Geroge J Hutton report grants, personal fees from Sanofi, grants, personal fees from Biogen, grants, personal fees from Novartis, grants, personal fees from Genentech, personal fees from Celgene, grants from MedImmune, grants from Adamas, during the conduct of the study, and grants from Biogen, Novartis, MedImmune, Hoffman-LaRoche, EMD Serono, Sanofi, and Mallinckrodt, and personal fees from Novartis, Sanofi, Celgene, outside the submitted work. Rajender R Aparasu reports grants from Incyte, Novartis, and Astellas outside the submitted work. The aforementioned authors report no other potential conflicts of interest for this work. The remaining authors report no conflicts of interest.

\section{References}

1. Williams MJ, Johnson K, Trenz HM, et al. Adherence, persistence, and discontinuation among Hispanic and African American patients with multiple sclerosis treated with fingolimod or glatiramer acetate. Curr Med Res Opin. 2018;34(1):107-115. doi:10.1080/03007995.2017. 1374937 
2. Remington G, Rodriguez Y, Logan D, Williamson C, Treadaway K. Facilitating medication adherence in patients with multiple sclerosis. Int J MS Care. 2013;15(1):36-45. doi:10.7224/1537-2073.2011-038

3. Erbay O, Usta Yesilbalkan O, Yuceyar N. Factors affecting the adherence to disease modifying therapy in patients with multiple sclerosis. J Neurosci Nurs. 2018;50(5):291-297. doi:10.1097/JNN.0000000000000395

4. Maroney M, Hunter SF. Implications for multiple sclerosis in the era of the Affordable Care Act: a clinical overview. Am J Manag Care. 2014;20(11 Suppl):S220-227.

5. Menzin J, Caon C, Nichols C, White LA, Friedman M, Pill MW. Narrative review of the literature on adherence to disease modifying therapies among patients with multiple sclerosis. J Manage Care Pharm. 2013;19(1 Supp A):S24-S40. doi:10.18553/jmcp.2013.19.s1.S24

6. Palmer L, Abouzaid S, Shi N, et al. Impact of patient cost sharing on multiple sclerosis treatment. Am J Pharm Benefits. 2012;4(Special Issue):SP28-SP36.

7. Patti F. Optimizing the benefit of multiple sclerosis therapy: the importance of treatment adherence. Patient Prefer Adherence. 2010;4:1-9. doi:10.2147/PPA.S8230

8. Higuera L, Carlin CS, Anderson S. Adherence to disease modifying therapies for multiple sclerosis. J Manage Care Spec Pharm. 2016;22 (12):1394-1401. doi:10.18553/jmcp.2016.22.12.1394

9. Klauer T, Zettl UK. Compliance, adherence, and the treatment of multiple sclerosis. J Neurol. 2008;255(Suppl 6):87-92. doi:10.1007/ s00415-008-6016-8

10. Hao J, Pitcavage J, Jones JB, Hoegerl C, Graham J. Measuring adherence and outcomes in the treatment of patients with multiple sclerosis. J Am Osteopath Assoc. 2017;117(12):737-747.

11. Longbrake EE, Cross AH, Salter A. Efficacy and tolerability of oral versus injectable disease modifying therapies for multiple sclerosis in clinical practice. Mult Scler J Exp Transl Clin. 2016;2.

12. Desai RJ, Mahesri M, Gagne JJ, et al. Utilization patterns of oral disease modifying drugs in commercially insured patients with multiple sclerosis. J Manag Care Spec Pharm. 2019;25(1):113-121.

13. Burks J. Adherence to disease modifying therapies and its impact on relapse, health resource utilization, and costs among patients with multiple sclerosis. - PubMed - NCBI. 2018.

14. Munsell M, Frean M, Menzin J, Phillips AL. An evaluation of adherence in patients with multiple sclerosis newly initiating treatment with a self-injectable or an oral disease modifying drug. Patient Prefer Adherence. 2017;11:55-62. doi:10.2147/PPA.S118107

15. Dionne C, G R, C A, C C. Abstract: Do Oral Disease Modifying Agents (DMTs) Improve Adherence to MS Treatment? A Comparison of Oral and Injectable Drugs (2015 CMSC Annual Meeting). https://cmsc.confex.com/cmsc/2015/webprogram/ Paper3275.html. 2015.

16. Sabido-Espin M, Munschauer R. Reasons for discontinuation of subcutaneous interferon beta-1a three times a week among patients with multiple sclerosis: a real-world cohort study. BMC Neurol. 2017;17(1):57.

17. Lanzillo R, Prosperini L, Gasperini C, et al. A multicentRE observational analysiS of PErsistenCe to Treatment in the new multiple sclerosis era: the RESPECT study. $J$ Neurol. 2018;265 (5):1174-1183. doi:10.1007/s00415-018-8831-x

18. Ferraro D, Camera V, Baldi E, et al. First-line disease modifying drugs in relapsing-remitting multiple sclerosis: an Italian real-life multicenter study on persistence. Curr Med Res Opin. 2018;34 (10):1803-1807. doi:10.1080/03007995.2018.1451311

19. Burks J, Marshall TS, Ye X. Adherence to disease modifying therapies and its impact on relapse, health resource utilization, and costs among patients with multiple sclerosis. Clinicoecon Outcomes Res. 2017;9:251-260.

20. Bergvall N, Petrilla AA, Karkare SU, et al. Persistence with and adherence to fingolimod compared with other disease modifying therapies for the treatment of multiple sclerosis: a retrospective US claims database analysis. J Med Econ. 2014;17(10):696-707. doi:10.3111/13696998.2014.940422
21. Agashivala N, Wu N, Abouzaid S, et al. Compliance to fingolimod and other disease modifying treatments in multiple sclerosis patients, a retrospective cohort study. BMC Neurol. 2013;13:9.

22. Bergvall N, Makin C, Lahoz R, et al. Comparative effectiveness of fingolimod versus interferons or glatiramer acetate for relapse rates in multiple sclerosis: a retrospective US claims database analysis. Curr Med Res Opin. 2013;29(12):1647-1656. doi:10.1185/03007995.2013.847411

23. Nazareth T, Friedman HS, Navaratnam P, et al. Persistency, medication prescribing patterns, and medical resource use associated with multiple sclerosis patients receiving oral disease modifying therapies: a retrospective medical record review. BMC Neurol. 2016;16(1):187.

24. Franklin JM, Krumme AA, Tong AY, et al. Association between trajectories of statin adherence and subsequent cardiovascular events. Pharmacoepidemiol Drug Saf. 2015;24(10):1105-1113. doi: $10.1002 /$ pds. 3787

25. Franklin JM, Shrank WH, Pakes J, et al. Group-based trajectory models: a new approach to classifying and predicting long-term medication adherence. Med Care. 2013;51(9):789-796.

26. Nagin DS. Group-based trajectory modeling: an overview. Ann Nutr Metab. 2014;65(2-3):205-210. doi:10.1159/000360229

27. Nicholas J, Edwards NC, Edwards RA, et al. ND3 Use of group-based trajectory modeling to identify adherence clusters in patients with multiple sclerosis newly-initiating once- or twice-daily oral disease modifying agents. Value Health. 2019;22:S43. doi:10.1016/j.jval.2019.04.061

28. Hansen L. The Truven Health MarketScan ${ }^{\circledR}$ Databases for Life Sciences Researchers. Ann Arbor: Truven Health Analytics; 2017.

29. Andersen R. Revisiting the behavioral model and access to medical care: does it matter? J Health Soc Behav. 1995;36(1):1-10. doi: $10.2307 / 2137284$

30. Truven Health MarketScan ${ }^{\circledR}$ Database.

31. Marrie RA. Comorbidity in multiple sclerosis: past, present and future. Clin Invest Med. 2019;42(1):E5-e12. doi:10.25011/cim. v42i1.32383

32. Marrie RA, Miller A, Sormani MP, et al. Recommendations for observational studies of comorbidity in multiple sclerosis. Neurology. 2016;86(15):1446-1453. doi:10.1212/WNL.0000000000002474

33. HCUP-US Tools \& Software Page. 2020. Available from: https:// www.hcup-us.ahrq.gov/toolssoftware/ccs/ccs.jsp.

34. Elixhauser A, Steiner C, Harris DR, Coffey RM. Comorbidity measures for use with administrative data. Med Care. 1998;36(1):8-27. doi:10.1097/00005650-199801000-00004

35. Quan H, Sundararajan V, Halfon P, et al. Coding algorithms for defining comorbidities in ICD-9-CM and ICD-10 administrative data. Med Care. 2005;43(11):1130-1139. doi:10.1097/01.mlr.0000182534.19832.83

36. Ontaneda D, Nicholas J, Carraro M, et al. Comparative effectiveness of dimethyl fumarate versus fingolimod and teriflunomide among MS patients switching from first-generation platform therapies in the US. Mult Scler Relat Disord. 2019;27:101-111. doi:10.1016/j.msard.2018. 09.038

37. Pyenson B, Tomicki S. Multiple Sclerosis: New Perspectives on the Patient Journey: 2019 Update. NY: Milliman Inc.; 2019.

38. Nicholas J, Boster A, Wu N, et al. Comparison of disease modifying therapies for the management of multiple sclerosis: analysis of healthcare resource utilization and relapse rates from US insurance claims data. PharmacoEconomics Open. 2018;2(1):31-41. doi:10.1007/s41669-017-0035-2

39. Vadhariya A, Fleming ML, Johnson ML, et al. Group-based trajectory models to identify sociodemographic and clinical predictors of adherence patterns to statin therapy among older adults. Am Health Drug Benefits. 2019;12(4):202-211.

40. Hess LM, Raebel MA, Conner DA, Malone DC. Measurement of adherence in pharmacy administrative databases: a proposal for standard definitions and preferred measures. Ann Pharmacother. 2006;40 (7-8):1280-1288. doi:10.1345/aph.1H018 
41. Martin BC, Wiley-Exley EK, Richards S, Domino ME, Carey TS, Sleath BL. Contrasting measures of adherence with simple drug use, medication switching, and therapeutic duplication. Ann Pharmacother. 2009;43(1):36-44. doi:10.1345/aph.1K671

42. Pednekar PP, Agh T, Malmenäs M, et al. Methods for measuring multiple medication adherence: a systematic review-report of the ISPOR medication adherence and persistence special interest group Value Health. 2019;22(2):139-156. doi:10.1016/j.jval.2018.08.006

43. Agashivala N, Wu N, Abouzaid S, et al. Compliance to fingolimod and other disease modifying treatments in multiple sclerosis patients, a retrospective cohort study. BMC Neurol. 2013;13:138. doi:10.1186/ 1471-2377-13-138

44. Alhazami M, Pontinha VM, Patterson JA, Holdford DA. Medication adherence trajectories: a systematic literature review. J Manage Care Spec Pharm. 2020;26(9):1138-1152. doi:10.18553/jmcp.2020.26.9.1138

45. Nagin DS. Group-based trajectory modeling: an overview. In: Handbook of Quantitative Criminology. Springer; 2010:53-67.

46. Nagin D, Tremblay R. Analyzing developmental trajectories of distinct but related behaviors: a group-based method. Psychol Methods. 2001;6(1):18-34. doi:10.1037/1082-989X.6.1.18

47. Arrandale V, Koehoorn M, MacNab Y, Kennedy MS How to use SAS ${ }^{\circledR}$ Proc Traj and SAS ${ }^{\circledR}$ Proc Glimmix in Respiratory Epidemiology. 2006.
48. Wabe N, Wojciechowski J, Wechalekar MD, et al. Disease activity trajectories in early rheumatoid arthritis following intensive DMARD therapy over 3 years: association with persistence to therapy. Int J Rheum Dis. 2017;20(10):1447-1456. doi:10.1111/1756-185X.13184

49. Brookhart MA, Wyss R, Layton JB, Stürmer T. Propensity score methods for confounding control in nonexperimental research. Circ Cardiovasc Qual Outcomes. 2013;6(5):604-611.

50. Austin PC, Stuart EA. Moving towards best practice when using inverse probability of treatment weighting (IPTW) using the propensity score to estimate causal treatment effects in observational studies. Stat Med. 2015;34(28):3661-3679. doi:10.1002/sim.6607

51. Schulte PJ, Mascha EJ. Propensity score methods: theory and practice for anesthesia research. Anesth Analg. 2018;127(4):1074-1084. doi:10.1213/ANE.0000000000002920

52. Nicholas J, Ontaneda D, Carraro M, et al. Development of an algorithm to identify Multiple Sclerosis (MS) disease severity based on healthcare costs in a US Administrative Claims Database (P2. 052). $A A N$ Enterprises. 2017.
Patient Preference and Adherence

\section{Publish your work in this journal}

Patient Preference and Adherence is an international, peer-reviewed, open access journal that focusing on the growing importance of patient preference and adherence throughout the therapeutic continuum. Patient satisfaction, acceptability, quality of life, compliance, persistence and their role in developing new therapeutic modalities and compounds to optimize clinical outcomes for existing disease

\section{Dovepress}

states are major areas of interest for the journal. This journal has been accepted for indexing on PubMed Central. The manuscript management system is completely online and includes a very quick and fair peer-review system, which is all easy to use. Visit http:// www.dovepress.com/testimonials.php to read real quotes from published authors. 